\title{
Extension of local-type inequality for the higher order correlation functions
}

\author{
Teruaki Suyama ${ }^{1}$ and Shuichiro Yokoyama ${ }^{2}$ \\ ${ }^{1}$ Research Center for the Early Universe, Graduate School of Science, The University of \\ Tokyo, Tokyo 113-0033, Japan \\ ${ }^{2}$ Department of Physics and Astrophysics, Nagoya University, Aichi 464-8602, Japan
}

\begin{abstract}
For the local-type primordial perturbation, it is known that there is an inequality between the bispectrum and the trispectrum. By using the diagrammatic method, we develop a general formalism to systematically construct the similar inequalities up to any order correlation function. As an application, we explicitly derive all the inequalities up to six and eight-point functions.
\end{abstract}




\section{Introduction}

Primordial non-Gaussianity has been attracting attention as a powerful probe to discriminate many existing inflation models (for example, see [1, 2]). Statistics of zero-mean Gaussian fluctuations can be characterized by a variance which corresponds to two-point function. Hence, the non-Gaussianity of fluctuations can be linked to the higher-order correlation functions. A lot of studies have been done for the bispectrum, i.e. three-point function of the primordial perturbations. For the so-called local type perturbation, the bispectrum is completely characterized by a single non-linearity parameter $f_{\mathrm{NL}}[3$. The current bound on $f_{\mathrm{NL}}$ is $-10<f_{\mathrm{NL}}<74$ at $95 \%$ confidence level [4].

In the last few years, trispectrum is becoming an important observable as well. For the local type non-Gaussianity, the trispectrum is specified by two non-linearity parameters $\tau_{\mathrm{NL}}$ and $g_{\mathrm{NL}}$ [5, 6]. The current bounds on $\tau_{\mathrm{NL}}$ and $g_{\mathrm{NL}}$ are respectively $-0.6<\tau_{\mathrm{NL}} / 10^{4} \leq$ 3.3 and $-7.4<g_{\mathrm{NL}} / 10^{5}<8.2$ at $95 \%$ confidence level [7]. An interesting fact that boosts the importance of the study of the trispectrum is that $\tau_{\mathrm{NL}}$ has a minimum determined by $f_{\mathrm{NL}}[8]$,

$$
\tau_{\mathrm{NL}} \geq \frac{36}{25} f_{\mathrm{NL}}^{2}
$$

It was shown in Ref. [9] that Planck satellite can measure $\tau_{\mathrm{NL}}$ down to 560. Therefore, future detection of $f_{\mathrm{NL}} \simeq 30$ which is the central value of the current bound implies that we should also detect non-vanishing $\tau_{\mathrm{NL}}$. Detection of both $f_{\mathrm{NL}}$ and $\tau_{\mathrm{NL}}$ (and possibly $g_{\mathrm{NL}}$ as well) enables us to constrain or even pin down the inflation model and the origin of the primordial fluctuations. In fact, in Ref. [7] the authors have discussed an observational constraint on the ratio between $f_{\mathrm{NL}}$ and $\tau_{\mathrm{NL}}$ which is defined as $A_{\mathrm{NL}} \equiv \tau_{\mathrm{NL}} /\left(6 f_{\mathrm{NL}} / 5\right)^{2}$.

There exist a few studies that extend the inequality (10). The authors of Refs. [10, 11] considered one-loop corrections to (1) and found the one-loop effect appears as the scale dependent modification of the coefficient in front of $f_{\mathrm{NL}}^{2}$. In Ref. [12, we have discussed that how many parameters one needs in order to characterize higher order correlation functions of primordial curvature fluctuations and also introduced the non-linearity parameters for the five-point function. The non-linearity parameters up to the six-point function were introduced in [13, 14] and two inequalities between the six- and four-point function were derived [13].

A potential importance and usefulness of the higher order correlation functions motivate us to look for the similar inequalities like (1) for the higher order correlation functions. In this paper, we provide a general formalism to construct the inequalities among the nonlinearity parameters for the higher order correlation functions. To this end, we will adopt the diagrammatic approach developed in Refs. [12, 15] that turns out to be a convenient way to study the higher order correlation functions. By using this method, in principle, we can systematically derive all the inequalities up to any-point function.

This paper is organized as follows. In the next section, we briefly review the higher order correlation functions of primordial curvature fluctuations, based on $\delta N$ formalism and the diagrammatic approach. In section 3 , we define the non-linearity parameters to 
characterize the higher order functions and construct the series of inequalities by applying the Cauchy-Schwarz inequality. We also present all inequalities up to the six- and eightpoint functions. Section 4 is spent on the conclusion of this paper.

\section{Higher order correlation functions of $\delta N$}

According to the $\delta N$ formalism [16, 17, 18, 19, the curvature perturbation on the uniform energy density hypersurface on super-horizon scales at $t=t_{f}$ is equal to the perturbation of the e-folding number evaluated from the flat hypersurface at $t=t_{*}$ to the uniform energy density hypersurface at $t_{f}$ at the same point:

$$
\zeta\left(t_{f}, \vec{x}\right)=\delta N\left(t_{f}, t_{*} ; \vec{x}\right) .
$$

The initial time $t_{*}$ can be chosen arbitrary. If we chose $t_{*}$ as the time slightly after the scale we are interested in leaves the Hubble horizon during inflation, then $\delta N$ in Eq. (22) is sourced by the scalar field fluctuations. Thus, $\zeta$ can be Taylor-expanded in terms of the scalar field fluctuations as

$$
\zeta\left(t_{f}, \vec{x}\right)=\sum \frac{1}{n !} N_{a_{1} a_{2} \cdots a_{n}} \delta \phi^{a_{1}}\left(t_{*}, \vec{x}\right) \cdots \delta \phi^{a_{n}}\left(t_{*}, \vec{x}\right),
$$

where $a_{1}, a_{2}, \cdots$ run from 1 to $p$ ( $p$ is a number of light scalar fields that acquire superhorizon scale fluctuations during inflation). In the following, we assume that $\delta \phi^{a}$ are Gaussian variables.

The two-point function of $\zeta$ can be written as

$$
\left\langle\zeta_{\vec{k}_{1}} \zeta_{\vec{k}_{2}}\right\rangle=(2 \pi)^{3} \delta\left(\vec{k}_{1}+\vec{k}_{2}\right) P_{\zeta}\left(k_{1}\right) .
$$

To leading order in the field fluctuations, $P_{\zeta}(k)$ is given by

$$
P_{\zeta}(k)=N_{a} N_{a} P(k), \quad P(k)=\frac{2 \pi^{2}}{k^{3}}\left(\frac{H_{*}}{2 \pi}\right)^{2},
$$

where summation over $a$ is assumed.

The three-point function of $\zeta$ can be written as

$$
\left\langle\zeta_{\vec{k}_{1}} \zeta_{\vec{k}_{2}} \zeta_{\vec{k}_{3}}\right\rangle=(2 \pi)^{3} \delta\left(\vec{k}_{1}+\vec{k}_{2}+\vec{k}_{3}\right) B_{\zeta}\left(k_{1}, k_{2}, k_{3}\right) .
$$

To leading order in the field fluctuations, $B_{\zeta}$ is given by

$$
B_{\zeta}\left(k_{1}, k_{2}, k_{3}\right)=\frac{N_{a} N_{b} N_{a b}}{\left(N_{c} N_{c}\right)^{2}}\left(P_{\zeta}\left(k_{1}\right) P_{\zeta}\left(k_{2}\right)+2 \text { perms. }\right) .
$$

Following literatures, the constant coefficient in front of the square of the power spectrum is written as 20

$$
f_{\mathrm{NL}}=\frac{5}{6} \frac{N_{a} N_{b} N_{a b}}{\left(N_{c} N_{c}\right)^{2}} .
$$


The four-point function of $\zeta$ can be written as

$$
\left\langle\zeta_{\vec{k}_{1}} \zeta_{\vec{k}_{2}} \zeta_{\vec{k}_{3}} \zeta_{\vec{k}_{4}}\right\rangle=(2 \pi)^{3} \delta\left(\vec{k}_{1}+\vec{k}_{2}+\vec{k}_{3}+\vec{k}_{4}\right) T_{\zeta}\left(\vec{k}_{1}, \vec{k}_{2}, \vec{k}_{3}, \vec{k}_{4}\right) .
$$

To leading order in the field fluctuations, $T_{\zeta}$ is given by

$$
\begin{aligned}
T_{\zeta}\left(\vec{k}_{1}, \vec{k}_{2}, \vec{k}_{3}, \vec{k}_{4}\right)= & \frac{N_{a} N_{a b} N_{b c} N_{c}}{\left(N_{d} N_{d}\right)^{3}}\left(P_{\zeta}\left(k_{1}\right) P_{\zeta}\left(k_{12}\right) P_{\zeta}\left(k_{4}\right)+11 \text { perms. }\right) \\
& +\frac{N_{a} N_{b} N_{c} N_{a b c}}{\left(N_{d} N_{d}\right)^{3}}\left(P_{\zeta}\left(k_{1}\right) P_{\zeta}\left(k_{2}\right) P_{\zeta}\left(k_{3}\right)+3 \text { perms. }\right),
\end{aligned}
$$

where $k_{i j} \equiv\left|\vec{k}_{i}+\vec{k}_{j}\right|$. Note that there appear two distinct terms that exhibit different wavenumber dependence. As a consequence, we need two parameters to specify the trispectrum. Following literatures, the two constant parameters are defined by [6]

$$
\tau_{\mathrm{NL}}=\frac{N_{a} N_{a b} N_{b c} N_{c}}{\left(N_{d} N_{d}\right)^{3}}, \quad g_{\mathrm{NL}}=\frac{25}{54} \frac{N_{a} N_{b} N_{c} N_{a b c}}{\left(N_{d} N_{d}\right)^{3}} .
$$

We can further proceed to higher order correlation functions as follows. The leading order of the $n$-point function is given by a sum of several distinct terms which are products of $(n-1)$ power spectra and exhibit different wavenumber dependence. According to the diagrammatic method [12], each of these leading terms has a corresponding connected tree diagram that consists of $n$ vertices and $(n-1)$ lines connecting two vertices. Tree diagrams for the two-, three- and four-point functions are shown in Fig. 1. In reverse, given a tree diagram with $n$ vertices, we can reconstruct the corresponding term that constitutes the $n$-point function as follows. First, we assign a different wavenumber $\left\{\vec{k}_{1}, \cdots, \vec{k}_{n}\right\}$ to each vertex of the diagram, where $\left\{\vec{k}_{1}, \cdots, \vec{k}_{n}\right\}$ are the arguments of the $n$-point function with the constraint $\vec{k}_{1}+\cdots+\vec{k}_{n}=0$. Next, we assign a wavenumber to each line in the diagram, too. Removing a line from the diagram yields two respectively connected sub-diagrams. Then, one assigns to the removed line the sum of the vectors associated with all vertices in one of the two sub-diagrams. Any use of the two sub-diagrams yields the same answer because of the constraint $\vec{k}_{1}+\cdots+\vec{k}_{n}=0$.

After associating the wavenumbers with all lines, we can assign the corresponding factors to the vertices and the lines. As for the vertex with $\mathrm{p}$ lines attached, assign the factor $N_{a 1 \cdots a_{p}}$ to it. As for the lines, assign $P$, where the argument of $P$ is set to the length of the wavenumber associated with each line. By multiplying all these factors assigned to vertices and lines, we obtain the corresponding leading term. Any term constructed in this way is given by a product of two terms. One is the constant term which is given by contracting all the pairs of indices of the expansion coefficients assigned to each vertex of the tree diagram. The other term is the $(n-1)$ product of the power spectra of the scalar field plus its permutations. If we replace each power spectrum of the scalar fields by that of the curvature perturbation, i.e. $P \rightarrow P_{\zeta} /\left(N_{a} N_{a}\right)$, the constant part gets a factor $\left(N_{a} N_{a}\right)^{n-1}$ in the denominator. Following the cases for the bispectrum and 


\section{2-point}

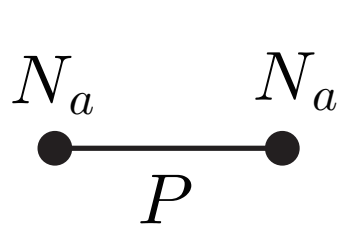

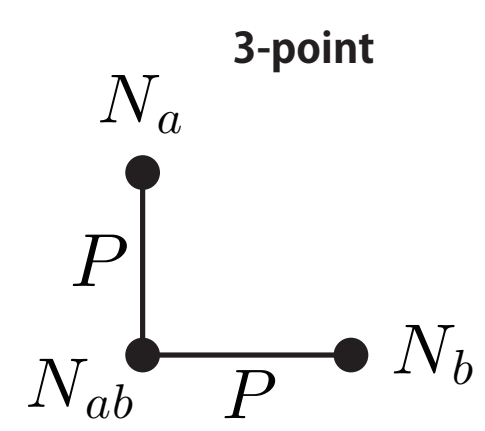

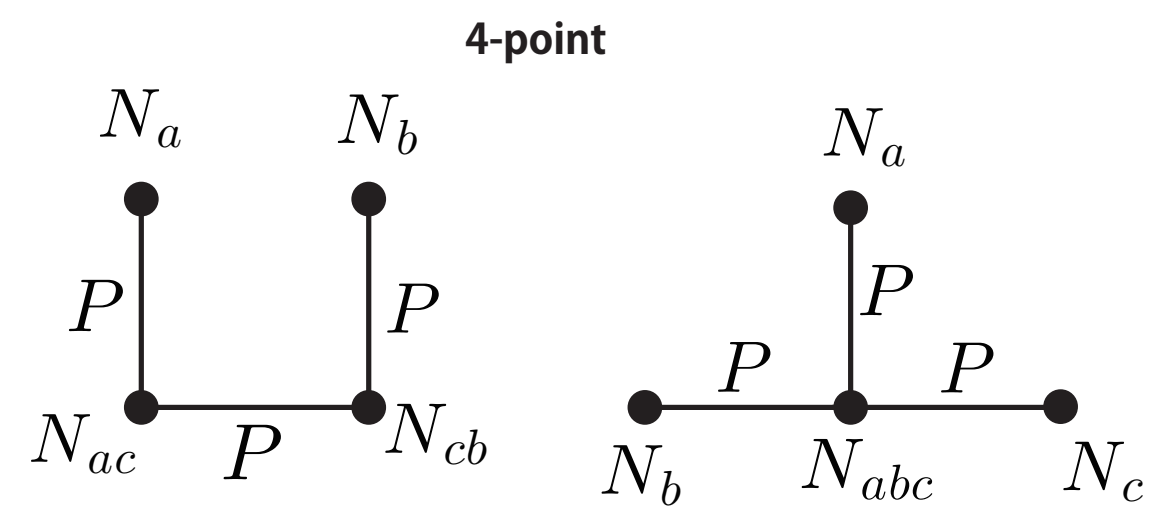

Figure 1: The tree diagrams corresponding to the power spectrum, bispectrum and trispectrum, based on $\delta N$ formalism. We find that we need two parameters in order to characterize the trispectrum.

the trispectrum, we define the constant parameter corresponding to this diagram as a coefficient in front of the $(n-1)$ product of $P_{\zeta}$. By taking the sum over all the possible (connected) tree diagrams with $n$ vertices, we obtain the $n$-point function.

It was shown in Ref. 12] that the functions constructed from two tree diagrams that are not isomorphic each other always yield different wavenumber dependence. Therefore, the number of independent constant parameters required to specify the $n$-point function is equal to the one of all the possible connected tree diagrams with $n$ vertices that are not isomorphic to each other.

A formula for a number $t_{n}$ of the independent connected tree diagrams with $n$ vertices is given in Refs. [21, 22]. According to Refs. [21, 22], we can formally construct a function $t(x)$ as an infinite series,

$$
t(x)=\sum_{n} t_{n} x^{n} .
$$


Then $t(x)$ is given by

$$
t(x)=r(x)-\frac{1}{2} r^{2}(x)+\frac{1}{2} r\left(x^{2}\right),
$$

where $r(x)$ is a function that satisfies,

$$
r(x)=x \exp \left[\sum_{k=1} \frac{1}{k} r\left(x^{k}\right)\right] .
$$

From the last equation, we can recursively obtain the Taylor-expansion coefficients of $r(x)$. Then from Eq. (13), we can recursively obtain $t_{n}$ as well. For example, $t_{2}=1, t_{3}=1, t_{4}=$ $2, t_{5}=3, t_{6}=6, t_{7}=11, t_{8}=23, \cdots$.

\section{Inequalities of the Cauchy-Schwarz type}

As we mentioned in the last section, wavenumber dependence of the $n$-point function is completely specified by a set of constant parameters each of which has a corresponding connected tree diagram with $n$ vertices. For the purpose of constructing the general inequalities among the non-linearity parameters, it is convenient to adopt a normalization condition,

$$
N_{a} N_{a}=1 .
$$

Without a loss of generality, this condition can be always imposed by rescaling the scalar field fluctuation. With this condition, the denominator of the non-linearity parameters for the $n$-point function, which is given by $\left(N_{a} N_{a}\right)^{n-1}$, becomes unity.

\subsection{Definition of the non-linearity parameters}

For any connected tree diagram with $n$ vertices, if we cut a line appearing in the diagram, the diagram splits into two sub-diagrams. If the resulting two sub-diagrams are isomorphic to each other, we call the parent diagram symmetric diagram. Obviously, in order for a diagram to be a symmetric diagram, $n$ must be even. Let us denote by $\kappa_{2 m}$ a number of all the symmetric diagrams among $t_{2 m}$ tree diagrams with $2 m$ vertices. For example, $\kappa_{2}=1, \kappa_{4}=1, \kappa_{6}=2, \kappa_{8}=4, \kappa_{10}=9, \cdots$. Our aim is to construct the inequalities of the Cauchy-Schwarz type for the non-linearity parameters up to the $n$-point functions. As it will be clear later, this is possible when $n$ is even. For the case of odd $n$, we can not construct the inequalities. For example, there are inequalities among the non-linearity parameters up to the six-point function. But there are no inequalities in which the highest order of the non-linearity parameters is five.

Since whether $n$ is even or odd is important, let us first write the non-linearity parameters for the $2 m$-point function as

$$
\left\{F_{2 m}^{(1)}, \cdots, F_{2 m}^{\left(t_{2 m}\right)}\right\}=\left\{\left\{\tau_{2 m}^{(1)}, \cdots, \tau_{2 m}^{\left(\kappa_{2 m}\right)}\right\},\left\{g_{2 m}^{(1)}, \cdots, g_{2 m}^{\left(t_{2 m}-\kappa_{2 m}\right)}\right\}\right\} .
$$




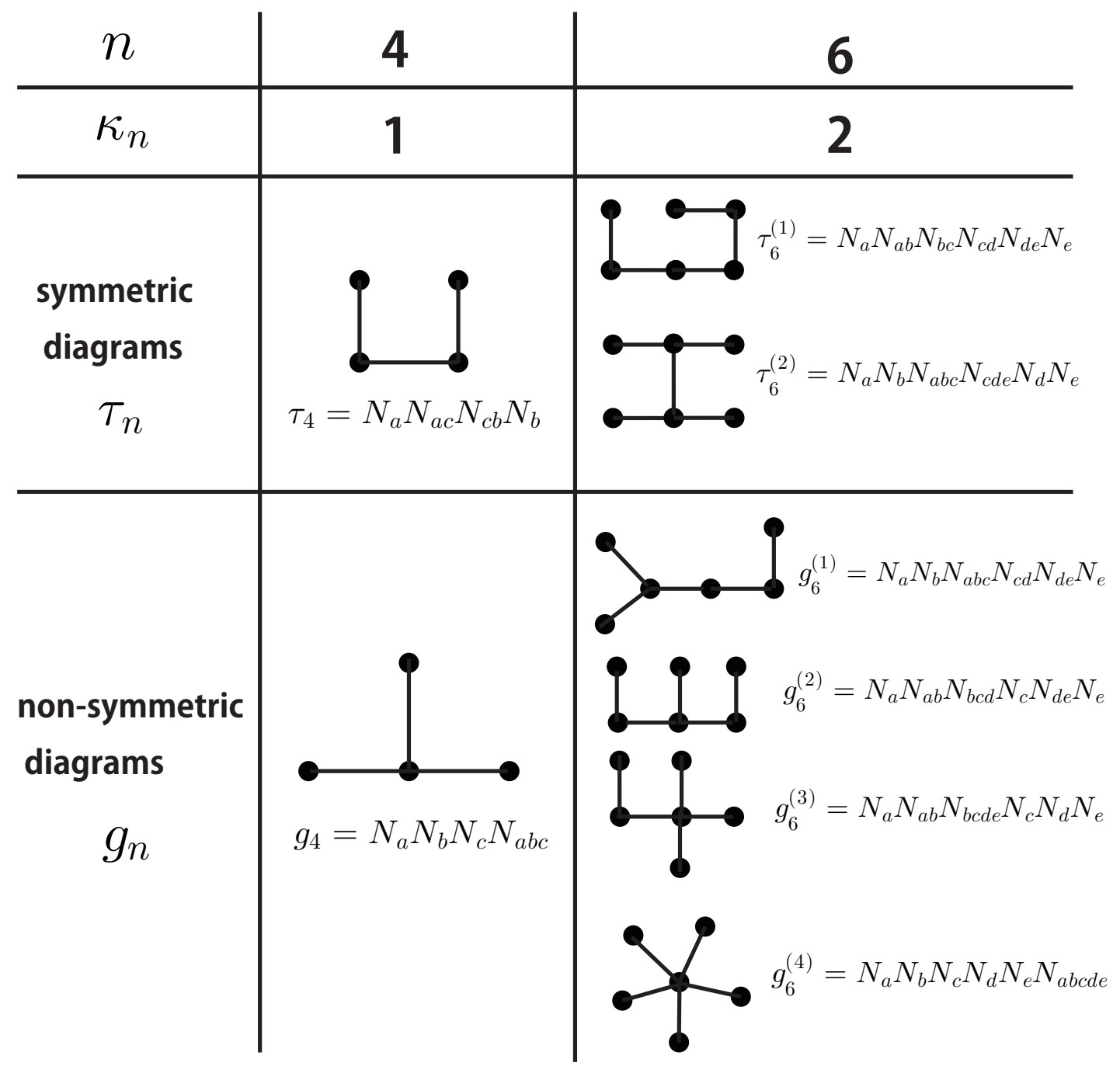

Figure 2: The symmetric and non-symmetric diagrams for $n=4,6$ cases. 


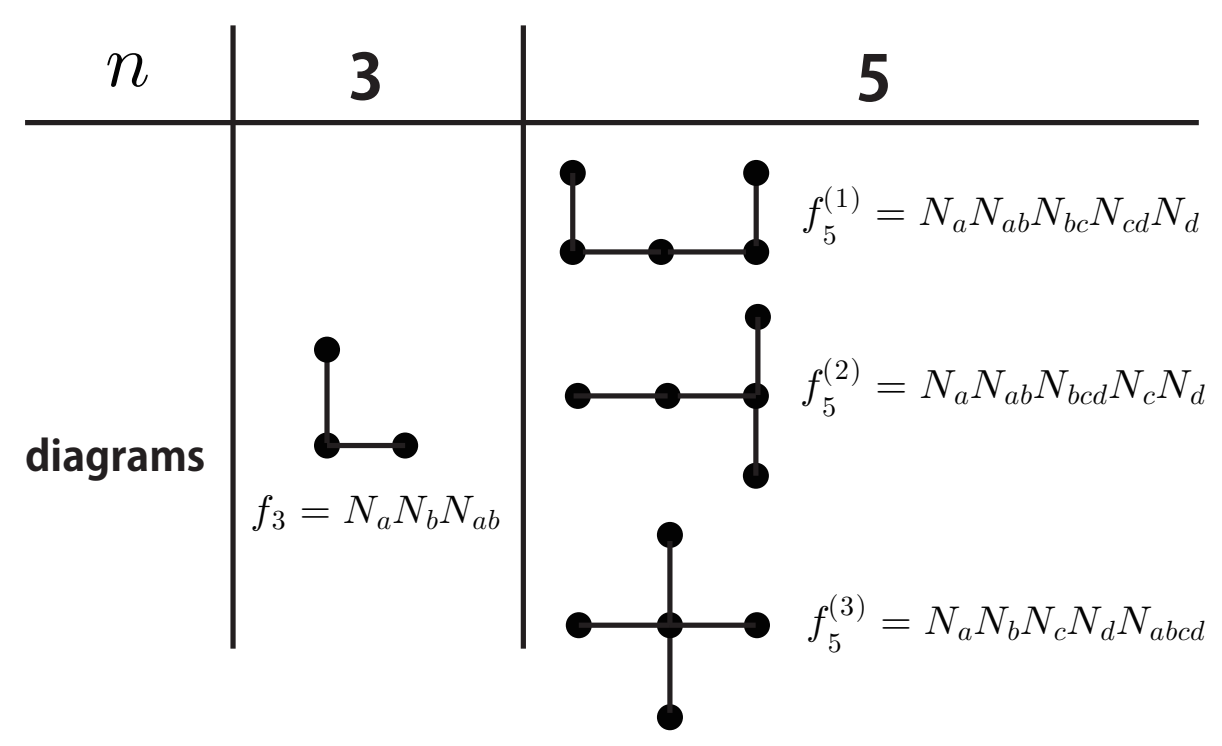

Figure 3: The diagrams for $n=3,5$ cases.

Here, $\tau_{2 m}^{(i)}$ and $g_{2 m}^{(i)}$ are the non-linearity parameters for the symmetric diagram and the non-symmetric diagram, respectively. In Fig. 2 , we show the symmetric diagrams and the non-symmetric diagrams for $n=4,6$-point functions. For the four-point function, a diagram corresponding to $\tau_{\mathrm{NL}}$ is a symmetric diagram while the one corresponding to $g_{\mathrm{NL}}$ is a non-symmetric diagram. Therefore, relations between $\left(\tau_{4}^{(1)}, g_{4}^{(1)}\right)$ and the conventional non-linearity parameters $\tau_{\mathrm{NL}}, g_{\mathrm{NL}}$ are given by

$$
\tau_{4}^{(1)}=\tau_{\mathrm{NL}}, \quad g_{4}^{(1)}=\frac{54}{25} g_{\mathrm{NL}} .
$$

For $(2 m-1)$-point function, we write the non-linearity parameters as

$$
\left\{F_{2 m-1}^{(1)}, \cdots, F_{2 m}^{\left(t_{2 m-1}\right)}\right\}=\left\{f_{2 m-1}^{(1)}, \cdots, f_{2 m-1}^{\left(t_{2 m-1}\right)}\right\} .
$$

In Fig. 3, we show the diagrams for $n=3,5$-point functions. A relation between $f_{3}^{(1)}$ and the conventional non-linearity parameter $f_{\mathrm{NL}}$ is given by

$$
f_{3}^{(1)}=\frac{6}{5} f_{\mathrm{NL}} \text {. }
$$

\subsection{Construction of the inequalities}

Let us suppose that the highest order of the non-linearity parameters appearing in the inequalities is $n=2 \mathrm{~m}$. We can construct the series of inequalities in a following way. 


\subsubsection{The highest order $n=2 m$}

Since $\tau_{2 m}^{(i)}$ is a non-linearity parameter corresponding to the symmetric diagram, it can be written as

$$
\tau_{2 m}^{(i)}=\vec{V}_{m}^{(i)} \cdot \vec{V}_{m}^{(i)},
$$

where $\vec{V}_{m}^{(i)}$ is a vector associated with a diagram $D_{m}^{(i)}$ which is one of the sub-diagrams generated by dividing the symmetric diagram into two diagrams that are isomorphic to each other. Therefore, $D_{m}^{(i)}$ is a tree diagram with $m$ vertices and with one external line. Next, let us consider a quantity

$$
\vec{V}_{m}^{(i)} \cdot \vec{V}_{m}^{(j)}
$$

for $i \neq j$. This must be a non-linearity parameter whose corresponding diagram is a diagram with $2 m$ vertices and is given by connecting the external line of $D_{m}^{(i)}$ with that of $D_{m}^{(j)}$. Since this diagram is a non-symmetric diagram, there exists some $k$ such that

$$
\vec{V}_{m}^{(i)} \cdot \vec{V}_{m}^{(j)}=g_{2 m}^{(k)} .
$$

Applying the Cauchy-Schwarz inequality to Eqs. (20) and (22) yields an inequality,

$$
\tau_{2 m}^{(i)} \tau_{2 m}^{(j)} \geq\left(g_{2 m}^{(k)}\right)^{2}
$$

A number of the independent inequalities of this kind is $\kappa_{2 m}\left(\kappa_{2 m}-1\right) / 2$. These are the inequalities among the non-linearity parameters for $2 m$-point function.

\subsubsection{Next highest order $2 m-1$}

Let us then lower the order by one and consider a quantity,

$$
\vec{V}_{m}^{(i)} \cdot \vec{V}_{m-1}^{(j)}
$$

This is a non-linearity parameter whose corresponding diagram is obtained by connecting the external line of $D_{m}^{(i)}$ with that of $D_{m-1}^{(j)}$. Therefore, there exists some $k$ such that

$$
\vec{V}_{m}^{(i)} \cdot \vec{V}_{m-1}^{(j)}=f_{2 m-1}^{(k)} \cdot
$$

Meanwhile, since a quantity $\vec{V}_{m-1}^{(j)} \cdot \vec{V}_{m-1}^{(j)}$ is a non-linearity parameter whose corresponding diagram is obtained by connecting the external lines of the same two $D_{m-1}^{(j)}$, we have

$$
\vec{V}_{m-1}^{(j)} \cdot \vec{V}_{m-1}^{(j)}=\tau_{2(m-1)}^{(j)} .
$$

Applying again the Cauchy-Schwarz inequality to Eqs. (25) and (26) yields

$$
\tau_{2 m}^{(i)} \tau_{2(m-1)}^{(j)} \geq\left(f_{2 m-1}^{(k)}\right)^{2}
$$

A number of the independent inequalities of this kind is $\kappa_{2 m} \times \kappa_{2 m-2}$. These are the inequalities among the non-linearity parameters for $2 m, 2 m-1$ and $2 m-2$-point functions. 


\subsubsection{Next-to-next highest order $2 m-2$}

Let us further lower the order by one and consider a quantity

$$
\vec{V}_{m}^{(i)} \cdot \vec{V}_{m-2}^{(j)}
$$

This is a non-linearity parameter whose corresponding diagram is obtained by connecting the external line of $D_{m}^{(i)}$ with that of $D_{m-2}^{(j)}$. Since this diagram can be both symmetric or non-symmetric, we write it as

$$
\vec{V}_{m}^{(i)} \cdot \vec{V}_{m-2}^{(j)}=F_{2 m-2}^{(k)} .
$$

Applying the same reasoning as before, we obtain

$$
\tau_{2 m}^{(i)} \tau_{2(m-2)}^{(j)} \geq\left(F_{2 m-2}^{(k)}\right)^{2}
$$

A number of the independent inequalities of this kind is $\kappa_{2 m} \times \kappa_{2 m-4}$. These are the inequalities among the non-linearity parameters for $2 m, 2(m-1)$ and $2(m-2)$-point functions.

By repeating the above procedures until the order reduces to one, we can obtain all the Cauchy-Schwarz type inequalities that involve the non-linearity parameters for the $2 m$-point function as the highest order. In particular, at the final procedure, we end up with getting a set of inequalities:

$$
\tau_{2 m}^{(i)} \geq\left(f_{m+1}^{(k)}\right)^{2}
$$

A number of the independent inequalities of this kind is $\kappa_{2 m}$.

Combining all these results, a number $a_{2 m}$ of independent inequalities that involve the non-linearity parameters for the $2 m$-point function as the highest order is given by

$$
a_{2 m}=\kappa_{2 m}\left(\frac{1}{2}\left(\kappa_{2 m}-1\right)+\kappa_{2 m-2}+\kappa_{2 m-4}+\cdots+\kappa_{2}\right) .
$$

For example, $a_{4}=1, a_{6}=5, a_{8}=22, a_{10}=108, \cdots$.

\subsection{Application to four, six and eight-point functions}

\subsubsection{Case of four-point function}

There are two different tree diagrams for the four-point function. One of them is a symmetric diagram which corresponds to $\tau_{\mathrm{NL}}$ and the other one is a non-symmetric diagram corresponding to $g_{\mathrm{NL}}$. According to the general argument we developed in the last subsection, there is no inequality between $\tau_{\mathrm{NL}}$ and $g_{\mathrm{NL}}$ because $\kappa_{4}=1$. Then, let us lower the order by one. At this stage, we obtain one inequality

$$
\tau_{4} \geq f_{3}^{2}
$$


In terms of the conventional non-linearity parameters, this can be written as

$$
\tau_{\mathrm{NL}} \geq \frac{36}{25} f_{\mathrm{NL}}^{2}
$$

Since we have reduced to the lowest order, there are no other inequalities.

\subsubsection{Case of six-point function}

Since $\kappa_{6}=2$, there exists a single inequality among the non-linearity parameters for the six-point function:

$$
\tau_{6}^{(1)} \tau_{6}^{(2)} \geq\left(g_{6}^{(1)}\right)^{2} .
$$

where the correspondence between the diagrams and the non-linearity parameters is given in Fig. 2. By lowering the order by one, we obtain $\kappa_{6} \times \kappa_{4}=2$ inequalities:

$$
\tau_{6}^{(1)} \tau_{4} \geq\left(f_{5}^{(1)}\right)^{2}, \quad \tau_{6}^{(2)} \tau_{4} \geq\left(f_{5}^{(2)}\right)^{2} .
$$

Reducing further the order by one, we obtain $\kappa_{6} \times \kappa_{2}=2$ inequalities:

$$
\tau_{6}^{(1)} \geq \tau_{4}^{2}, \quad \tau_{6}^{(2)} \geq g_{4}^{2} .
$$

The last two inequalities were also provided in [13]. Since we have reduced to the lowest order, there are no other inequalities. Total number of inequalities is five.

\subsubsection{Case of eight-point function}

Since $\kappa_{8}=4$, there exist six inequalities among the non-linearity parameters for the eightpoint function:

$$
\begin{array}{ll}
\tau_{8}^{(1)} \tau_{8}^{(2)} \geq\left(g_{8}^{(1)}\right)^{2}, \quad \tau_{8}^{(1)} \tau_{8}^{(3)} \geq\left(g_{8}^{(2)}\right)^{2}, \quad \tau_{8}^{(1)} \tau_{8}^{(4)} \geq\left(g_{8}^{(3)}\right)^{2}, \\
\tau_{8}^{(2)} \tau_{8}^{(3)} \geq\left(g_{8}^{(4)}\right)^{2}, \quad \tau_{8}^{(2)} \tau_{8}^{(4)} \geq\left(g_{8}^{(5)}\right)^{2}, \quad \tau_{8}^{(3)} \tau_{8}^{(4)} \geq\left(g_{8}^{(6)}\right)^{2} .
\end{array}
$$

where the correspondence between the diagrams and the non-linearity parameters is given

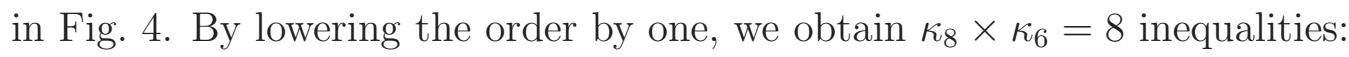

$$
\begin{aligned}
& \tau_{8}^{(1)} \tau_{6}^{(1)} \geq\left(f_{7}^{(1)}\right)^{2}, \quad \tau_{8}^{(1)} \tau_{6}^{(2)} \geq\left(f_{7}^{(2)}\right)^{2}, \quad \tau_{8}^{(2)} \tau_{6}^{(1)} \geq\left(f_{7}^{(3)}\right)^{2}, \quad \tau_{8}^{(2)} \tau_{6}^{(2)} \geq\left(f_{7}^{(4)}\right)^{2}, \\
& \tau_{8}^{(3)} \tau_{6}^{(1)} \geq\left(f_{7}^{(5)}\right)^{2}, \quad \tau_{8}^{(3)} \tau_{6}^{(2)} \geq\left(f_{7}^{(6)}\right)^{2}, \quad \tau_{8}^{(4)} \tau_{6}^{(1)} \geq\left(f_{7}^{(7)}\right)^{2}, \quad \tau_{8}^{(4)} \tau_{6}^{(2)} \geq\left(f_{7}^{(1)}\right)^{2},
\end{aligned}
$$

where the correspondence between the diagrams and the non-linearity parameters is given in Fig. [5. Reducing further the order by one, we obtain $\kappa_{8} \times \kappa_{4}=4$ inequalities:

$$
\tau_{8}^{(1)} \tau_{4} \geq\left(g_{6}^{(1)}\right)^{2}, \quad \tau_{8}^{(2)} \tau_{4} \geq\left(g_{6}^{(2)}\right)^{2}, \quad \tau_{8}^{(3)} \tau_{4} \geq\left(g_{6}^{(3)}\right)^{2}, \quad \tau_{8}^{(4)} \tau_{4} \geq\left(\tau_{6}^{(1)}\right)^{2} .
$$




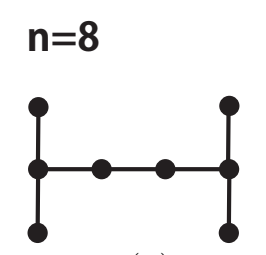

$\tau_{8}^{(1)}$

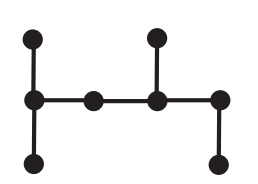

$g_{8}^{(1)}$

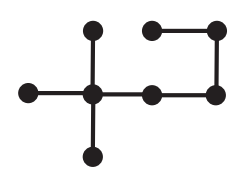

$g_{8}^{(6)}$

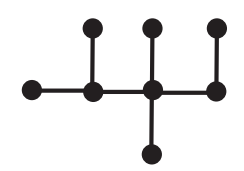

$g_{8}^{(11)}$

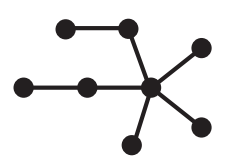

$g_{8}^{(16)}$
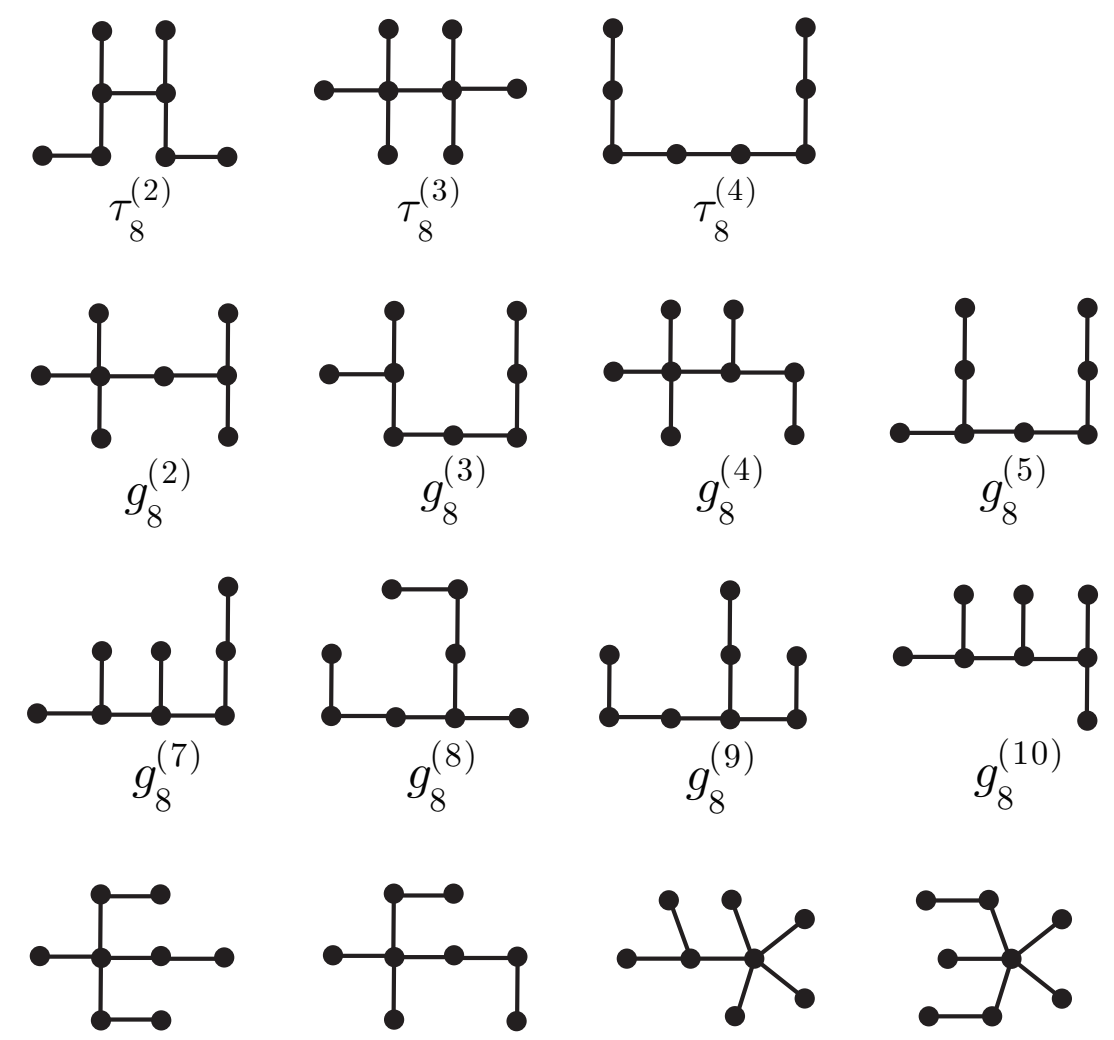

$g_{8}^{(14)}$
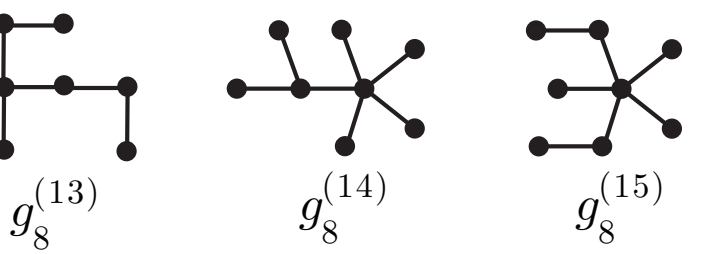

$g_{8}^{(15)}$
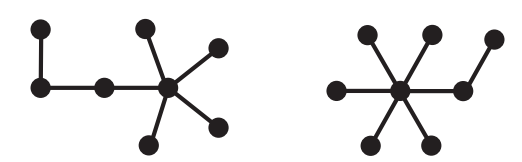

$g_{8}^{(17)}$

$g_{8}^{(18)}$

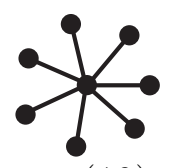

$g_{8}^{(19)}$

Figure 4: The correspondence between the diagrams and the non-linearity parameters for 8-point function. 


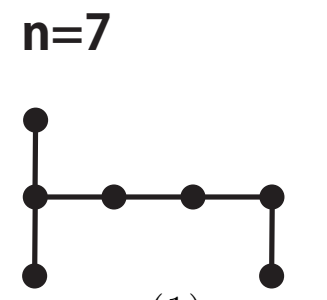

$f_{7}^{(1)}$

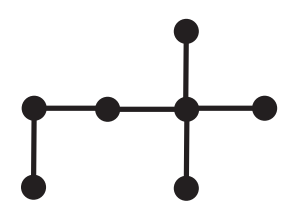

$f_{7}^{(5)}$

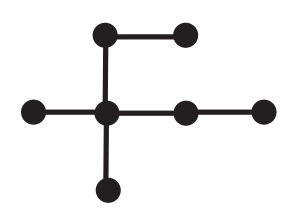

$f_{7}^{(9)}$
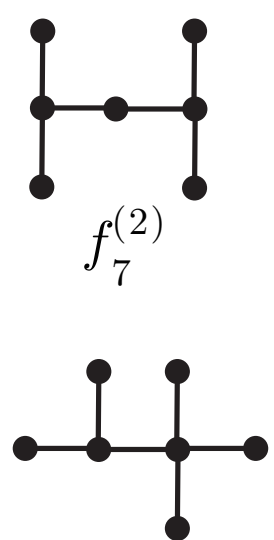

$f_{7}^{(6)}$

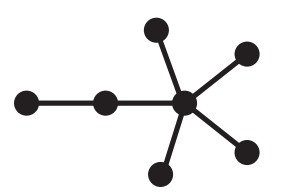

$f_{7}^{(10)}$
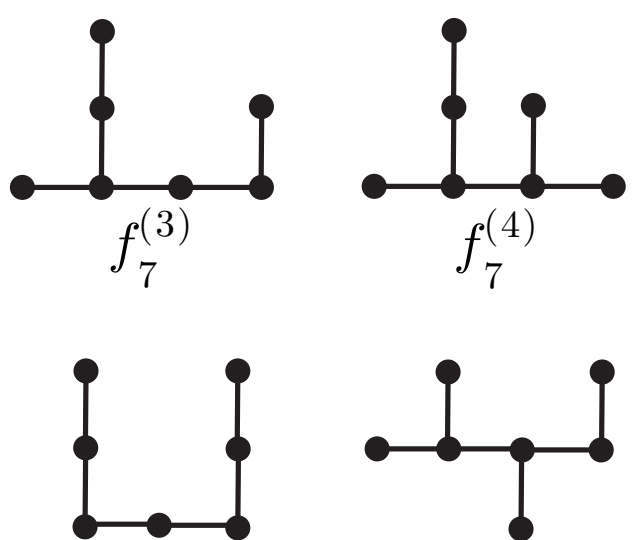

$f_{7}^{(7)}$

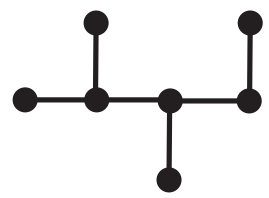

$f_{7}^{(8)}$

Figure 5: The correspondence between the diagrams and the non-linearity parameters for 7-point function. 
Reducing further the order by one, we obtain $\kappa_{8} \times \kappa_{2}=4$ inequalities:

$$
\tau_{8}^{(1)} \geq\left(f_{5}^{(2)}\right)^{2}, \quad \tau_{8}^{(2)} \geq\left(f_{5}^{(2)}\right)^{2}, \quad \tau_{8}^{(3)} \geq\left(f_{5}^{(3)}\right)^{2}, \quad \tau_{8}^{(4)} \geq\left(f_{5}^{(1)}\right)^{2} .
$$

Since we have reduced to the lowest order, there are no other inequalities. Total number of inequalities is 22 .

\section{Conclusion}

The primordial non-Gaussianity has been focused on by many authors as a new probe of the inflation dynamics. In addition to the bispectrum, use of the higher order correlation functions will become useful in the future. In particular, the inequality between $\tau_{\mathrm{NL}}$ and $g_{\mathrm{NL}}$ shows the importance of the trispectrum to look for the non-Gaussianity in the primordial perturbations.

In this paper, we developed a general formalism to construct the Cauchy-Schwarz type inequalities among the non-linearity parameters for the higher order correlation functions. This method enables us to derive all the inequalities up to any-point function. As an application, we explicitly derived all the inequalities up to the four, six and eight-point functions. We first confirmed that there is just one inequality, which is Eq. (11), up to the four-point function. Up to the six-point function, there are five new inequalities two of which were given in Ref. [13]. Up to the eight-point function, there are further 22 new inequalities.

Acknowledgments: T. S. is supported by a Grant-in-Aid for JSPS Fellows No. 1008477. S. Y. is partially supported by the Grant-in-Aid for Scientific research from the Ministry of Education, Science, Sports, and Culture, Japan, No. 22340056. S. Y. also acknowledges support from the Grant-in-Aid for the Global COE Program "Quest for Fundamental Principles in the Universe: from Particles to the Solar System and the Cosmos" from MEXT, Japan. The authors also thank the Yukawa Institute for Theoretical Physics at Kyoto University, where this work was discussed during the YITP-T-10-05 on "Cosmological Perturbation and Cosmic Microwave Background".

\section{References}

[1] E. Komatsu et al., arXiv:0902.4759 [astro-ph.CO].

[2] N. Bartolo, S. Matarrese and A. Riotto, arXiv:1001.3957 [astro-ph.CO].

[3] E. Komatsu and D. N. Spergel, Phys. Rev. D 63, 063002 (2001) arXiv:astro-ph/0005036.

[4] E. Komatsu et al. [WMAP Collaboration], Astrophys. J. Suppl. 192, 18 (2011) arXiv:1001.4538 [astro-ph.CO]]. 
[5] L. Boubekeur and D. H. Lyth, Phys. Rev. D 73, 021301 (2006) arXiv:astro-ph/0504046.

[6] C. T. Byrnes, M. Sasaki and D. Wands, Phys. Rev. D 74, 123519 (2006) arXiv:astro-ph/0611075.

[7] J. Smidt, A. Amblard, C. T. Byrnes, A. Cooray, A. Heavens and D. Munshi, Phys. Rev. D 81, 123007 (2010) [arXiv:1004.1409 [astro-ph.CO]].

[8] T. Suyama and M. Yamaguchi, Phys. Rev. D 77, 023505 (2008) arXiv:0709.2545 [astro-ph]].

[9] N. Kogo and E. Komatsu, Phys. Rev. D 73, 083007 (2006) arXiv:astro-ph/0602099].

[10] T. Suyama, T. Takahashi, M. Yamaguchi and S. Yokoyama, JCAP 1012, 030 (2010) arXiv:1009.1979 [astro-ph.CO]].

[11] N. S. Sugiyama, E. Komatsu and T. Futamase, arXiv:1101.3636 [gr-qc].

[12] S. Yokoyama, T. Suyama and T. Tanaka, JCAP 0902, 012 (2009) arXiv:0810.3053 [astro-ph]].

[13] C. Lin and Y. Wang, JCAP 1007, 011 (2010) arXiv:1004.0461 [astro-ph.CO]].

[14] J. Meyers and N. Sivanandam, arXiv:1104.5238 [astro-ph.CO].

[15] C. T. Byrnes, K. Koyama, M. Sasaki and D. Wands, JCAP 0711, 027 (2007) arXiv:0705.4096 [hep-th]].

[16] A. A. Starobinsky, JETP Lett. 42, 152 (1985) [Pisma Zh. Eksp. Teor. Fiz. 42, 124 (1985)].

[17] M. Sasaki and E. D. Stewart, Prog. Theor. Phys. 95, 71 (1996) arXiv:astro-ph/9507001.

[18] M. Sasaki and T. Tanaka, Prog. Theor. Phys. 99, 763 (1998) arXiv:gr-qc/9801017.

[19] D. H. Lyth, K. A. Malik and M. Sasaki, JCAP 0505, 004 (2005) arXiv:astro-ph/0411220.

[20] D. H. Lyth and Y. Rodriguez, Phys. Rev. Lett. 95, 121302 (2005) arXiv:astro-ph/0504045.

[21] J. Riordan, "Introduction to Combinatorial Analysis", Dover Publications, INC., Mineola, New York

[22] J. N. Fry, Astrophys. J. 279 (1984) 499. 